\title{
Infrared imaging and thermomechanical behaviour of solid materials
}

\author{
by A. Chrysochoos
}

\author{
Laboratoire de Mécanique et Génie Civil, UMR 5508 CNRS-UMII, CC081, Place E Bataillon \\ 34095 Montpellier cedex 05, France. Email : chryso@Imgc.univ-montp2.fr
}

\begin{abstract}
:
Infrared thermography gives valuable information useful to observe, understand and model the thermomechanical behaviour of solids. The theoretical framework of standard materials is used to define the different heat sources induced by a deformation process. These sources are estimated from infrared data by using the local heat equation. The image processing involves Fourier techniques and its reliability is studied using spectral methods. An example of thermomechanical analysis of a rubberlike material illustrates the great interest of infrared data for improving behaviour models.
\end{abstract}

\section{Introduction}

Mechanics of materials is nowadays a very active research field. It deals with the behaviour of solids. From a theoretical viewpoint, the behaviour is represented by a set of constitutive equations which supplement the balance relations (mass, movement, energy). From a more practical viewpoint, these equations are essential for engineers trying to optimise a stamping process, to design a mechanical part, to predict the life span of a component. Together with boundary conditions, the phenomenological equations represent the whole "physics" integrated into a computational code. Taking into consideration the constant efforts carried out by many research centres to extend the reliability of models [1,2], the point of view exposed hereafter consists in analysing the material behaviour both thermodynamically and mechanically [3,4].

The deformation of solid materials is accompanied by dissipative effects. Irreversibility, whose nature depends on the behaviour of the material, results in an intrinsic dissipation of energy due to plasticity, viscosity or damage. It may also be due to thermal dissipation induced by heat diffusion and generated by thermomechanical couplings associated with thermoelasticity, rubber elasticity or first-order phase transition. The temperature variations produced by these volume heat sources depend not only on the diffusivity of the material, but also on the boundary conditions. Thus, the thermal data are not completely intrinsic to the material behaviour. Techniques of infrared image processing were then investigated in order to take into account the heat diffusion and to estimate, under certain conditions, the distribution of heat sources during a deformation process.

After recalling the form of the energy balance, the numerical methods of data processing will be briefly presented. In the case of a homogeneous test, it is possible to draw up an energy balance useful for constructing behavioural models. As an example, the analysis of thermomechanical data extracted from tests performed on a rubberlike material will be finally proposed. The data analysis will show that the internal energy of such a material is a function of absolute temperature only, as soon as the rates of given-off heat and of mechanical work become equal. This result is a basic hypothesis of classical models of rubber elasticity and is 
generally justified by considerations at a molecular scale. Here it is experimentally evidenced at a continuum scale.

\section{Thermomechanical framework}

We work in the framework of classical thermodynamics of irreversible processes. It assumes the local state axiom [5,6]. Classically, we characterise the equilibrium of a volume element by the absolute temperature $T$, a strain tensor $\varepsilon$, and a vector $\alpha$ whose the $n$ scalar components complete the description of the equilibrium state.

If $\psi$ and $s$ denote respectively the specific Helmholtz free energy and the specific entropy, the Clausius-Duhem inequality, derived form of the second principle of thermodynamics, defines the dissipation $d$ as

$$
d=\sigma: \mathbf{D}-\rho \psi, \varepsilon: \dot{\varepsilon}-\rho \psi, \alpha \cdot \dot{\alpha}-\frac{\mathbf{q}}{T} \cdot \operatorname{grad} T \geq 0,
$$

where $\boldsymbol{\sigma}$ is the Cauchy stress tensor, $\mathbf{D}$ the Eulerian strain rate tensor, $\rho$ the mass density, $\mathbf{q}$ the heat influx vector. The dot stands for the material time derivative. The equality $d=0$ then characterises reversible thermodynamic processes. Classically, the intrinsic (mechanical) dissipation $d_{1}$ and the thermal dissipation $d_{2}$ are supposed to be separately positive. They are respectively defined by

$$
\begin{gathered}
d_{1}=\sigma: \mathbf{D}-\rho \psi, \varepsilon: \dot{\boldsymbol{\varepsilon}}-\rho \psi, \alpha \cdot \dot{\alpha} \geq 0, \\
d_{2}=-\frac{\mathbf{q}}{T} \cdot \operatorname{grad} T \geq 0 .
\end{gathered}
$$

Per unit volume, the intrinsic dissipation $d_{1}$ is the difference between the rate of deformation work $w_{\mathrm{ext}}^{\bullet}=\sigma: \mathbf{D}$ and the elastic and stored energy rates $w_{\mathrm{e}}^{\bullet}+w_{\mathrm{s}}^{\bullet}=\rho \psi_{, \varepsilon} \cdot \dot{\boldsymbol{\varepsilon}}+\rho \psi_{, \alpha} \cdot \dot{\alpha}$. The symbol ( ) means that the variation of () is path-dependent.

Deduced from both principles of thermodynamics, the local heat conduction equation reads

$$
\rho C \dot{T}+\operatorname{div} \mathbf{q}=d_{1}+\rho T \psi_{, \mathrm{T}}: \dot{\boldsymbol{\varepsilon}}+\rho T \psi_{, \mathrm{T} \alpha} \cdot \dot{\alpha}+r_{\mathrm{e}},
$$

where $C$ denotes the specific heat capacity while $r_{\mathrm{e}}$ symbolises the external heat supply. The intrinsic dissipation $d_{1}$ and the thermomechanical coupling terms $\rho T \Psi_{T,}: \dot{\varepsilon}$ and $\rho T \psi_{, T \alpha} \cdot \dot{\alpha}$ have been gathered in the right hand member of (4). For materials considered here, the Lord Kelvin's term $\rho T \psi_{, T}: \dot{\varepsilon}$ represents the volume heat rate due to thermoelastic couplings. Taking into account an isotropic conduction of heat $(\mathbf{q}=-k \operatorname{grad} T)$, we underline that the left side of Eq. (4) is a partial derivative operator applied to temperature. Its estimate leads to a local determination of heat sources.

\section{Experimental set-up and image processing}

The experimental set-up involves a $100 \mathrm{kN}$ tension-compression testing machine coupled with an infrared camera (Agema $880 \mathrm{sw}$ ). The lens axis of the camera is kept fixed and perpendicular to the sample surface[7]. The main object of the data processing is to derive the distribution of heat sources from surface temperature.

In what follows, the case, specific but important in practice, of thin and flat parallelepipedic samples will be considered. For such a simple geometry, the temperature gradients throughout the sample thickness are assumed to be small. The heat sources are then determined by evaluating the left side of Eq. (4) depth-wise averaged. A thermal image 
contains typically $256 \times 200$ pixels. The pixel surface is around $0.04 \mathrm{~mm}^{2}$ in the optical conditions of our tests. The thermal data being discrete and noisy, the differential operator is estimated by using low-pass filtering, properties of discrete Fourier transform and Fourier series [7]. The frame rate being relatively small (6 images per second), the data filtering is mainly performed spatially. To avoid parasitic frequencies due to the non-periodicity of images and to reduce the influence of boundary effects, here strongly amplified by the Laplacian estimate, a periodic extension of the data is performed at first. The thermal noise is well approximated by a white noise having a Gaussian probability distribution and a uniform power spectrum. This last feature implies that the filtering method, whatever it is, cannot eliminate completely the parasitic frequencies. Consequently, a calibration of the method in conditions close to the experimental situation is inevitable.

The check of the data processing is realised as follows : starting from a given heat source distribution, the corresponding temperature charts are calculated using spectral methods with realistic boundary conditions. The influence of the noise amplitude on the data processing is then tested. The heat sources reconstructed from the noisy thermal data are compared to the sources given beforehand. More information on the data processing and its performances can be found in [7]. As expected, the check of data processing shows that errors on source determination grow rapidly with the thermal noise amplitude. The unstable nature of the temperature-source passage (inverse problem) comes from the regularising effects of the diffusion phenomena (direct problem).

To limit noise effects, the cut-off frequencies of filters can be reduced; in return, one observes, as it does, a decrease of the sources intensity and a spatial sprawl of zones where the heat sources concentrate.

Besides, in the case of the experimental conditions, the following hypotheses are made:

- the specific heat capacity $C$ and the isotropic conduction coefficient $k$ are material constants independent of the internal state,

- the external heat supply $r_{\mathrm{e}}$ is time independent,

- the convective terms of the material time derivative are negligible.

These hypotheses are reasonable for many classical situations ; they may become widely unsound when strong anisotropy pre-exists or develops during the straining or when the thermomechanical loading leads to dynamic instabilities.

\section{An example of thermodynamic analysis}

Some polymers can undergo very large strains in a reversible way. This remarkable behaviour is often called rubber (or entropic) elasticity. The chosen example of behaviour analysis deals with one of these rubberlike materials: it is a thermoplastic polymer having a small density of crosslinks (grade 2533) and a constant mass density $\rho$.

In figure 1, the stress-strain hysteresis loops associated with increasing load-unload sequences is plotted. If $w_{\mathrm{ch}}^{\bullet}$ represents the overall heat rate, it is shown in [8] that the volume energy $A_{\mathrm{h}}$ related to hysteresis area can be written as

$$
A_{\mathrm{h}}=\oint_{C y c l e} w_{\mathrm{ch}}^{\bullet} \mathrm{d} \tau=\oint_{C y c l e}\left(d_{1}+\rho T \psi_{, T \varepsilon}: \dot{\varepsilon}+\rho T \psi_{, T \alpha} \cdot \dot{\alpha}\right) \mathrm{d} \tau,
$$

as soon as a hysteresis loop corresponds to a thermodynamic cycle. Both dissipation and coupling can then induce hysteresis mechanisms. Figure 2 shows that $w_{\mathrm{ch}}^{\bullet}$ is very often equal to the rate of mechanical energy $w_{\text {ext }}^{\bullet}=\sigma: \mathbf{D}$. 
To interpret this result, we write the local expression of the first principle of thermodynamics

$$
\rho \dot{e}=w_{\text {ext }}^{\bullet}-\operatorname{divq}+r_{e},
$$

where $e$ is the specific internal energy. By linking Eqs. (4) and (6), we obtain

$$
\dot{e}=C \dot{T} \text {. }
$$

This last result is the fundamental hypothesis of the molecular theory of rubber elasticity [xx]. The analogy with ideal gases leads to an internal energy independant of elongation, the stress being attributed to a so-called configuration entropy. As in classical elasticity, temperature $T$ and strain $\varepsilon$ are chosen as state variables and no intrinsic dissipation occurs. By virtue of Eq.(7), the free energy is of the form [9]

$$
\psi(T, \varepsilon)=T \xi(\varepsilon)+\eta(T)
$$

The stress is then proportional to the temperature

$$
\sigma(T, \varepsilon)=\rho \psi_{, \varepsilon}(T, \varepsilon)=\rho T \xi_{, \varepsilon}(\varepsilon)=\left(T / T_{0}\right) \sigma\left(T_{0}, \varepsilon\right)
$$

If the material is stress free at uniform temperature $T_{0}\left(\sigma\left(T_{0}, \varepsilon\right)=0\right)$, the material will remain stress free if the temperature is changed without deformation taking place. This result prohibits thermal expansion. In particular, the entropic elastic model is not able to predict the famous "thermoelastic" inversion effect. This property of rubberlike materials can be written in the case of tensile test as [10]

$$
\left\{\begin{array}{l}
(\partial \sigma / \partial T)_{\varepsilon}=\mathrm{n}(\varepsilon)<0 \text { if } \varepsilon<\varepsilon_{0} \\
(\partial \sigma / \partial T)_{\varepsilon}=\mathrm{p}(\varepsilon)>0 \text { if } \varepsilon>\varepsilon_{0}
\end{array}\right.
$$

This leads some authors to modify the classical scheme of entropic elasticity [9] assuming that a part of the internal energy is now function of strain.

We followed another way [11]. In Figure 2, thermoelastic effects can be observed at the beginning of the first loading. Moreover, a slight dissipation induces a drift of the energy signals throughout the test. As a consequence, we claim that entropic elasticity is always defined by Eq.(7) but we consider that the overall strain is not strictly due to entropic elasticity. This leads us to introduce a strain tensor $\varepsilon_{c}$ characterising the configuration of the polymer chain network as a new state variable. The free energy is then decomposed into thermelastic part and entropic elastic part so that we get

$$
\psi\left(T, \boldsymbol{\varepsilon}, \boldsymbol{\varepsilon}_{\mathrm{c}}\right)=\psi_{\text {the }}\left(T, \boldsymbol{\varepsilon}-\boldsymbol{\varepsilon}_{\mathrm{c}}\right)+T \xi\left(\boldsymbol{\varepsilon}_{\mathrm{c}}\right)+\eta(T)
$$

With Eq. (11), the thermoelastic inversion can be predicted (Figure 3). The change of slope corresponds to the change of dominating coupling phenomenon. It can be shown that for small strain, thermoelasticity remains preponderant and the slope $(\partial \sigma / \partial T)_{\varepsilon}$ is negative. For larger strain, the entropic elasticity dominates and induces a positive slope. Finally, a dissipation potential function of $\dot{\boldsymbol{\varepsilon}}_{\mathrm{c}}$ can be introduced to take account of irreversibility induced by the elongation of the polymer chains.

\section{Concluding comments}

The formalism of standard material gives a coherent and flexible theoretical framework. Its flexibility comes from the possibility of introducing state variables for summing up the various microscopic events which accompany the macroscopic deformation of materials. Moreover, this framework allows to define the different heat sources induced by deformation and a heat equation essential to link thermal and mechanical effects. In addition, infrared thermography represents a supplementary asset to check the validity of constitutive equations of models constructed within such a thermomechanical framework. The passage from temperature to 
heat source provides with information that is more intrinsic to material behaviour than temperature. At present, the tendency is to correlate the distributions of heat source with that of strain rate obtained by digital image correlation [12]. This dual experimental approach should facilitate the identification of constitutive equations in presence of localisation phenomena that frequently arise at large strain when damage develops within the material.

\section{References}

[1] LEMAITRE, J. and. CHABOCHE, J.-L., "Mécanique des Matériaux Solides", Dunod Paris, 1985.

[2] FRANÇOIS, D., PINEAU A. and ZAOUI, A., "Comportement mécanique des matériaux" , Vol.1 et 2, Hermes, 1991.

[3] CHRYSOCHOOS, A. and BELMAHJOUB, F., "Thermographic Analysis of Thermomechanical Couplings", p 55-68, Archives of Mechanics,44.1, 1992.

[4] CHRYSOCHOOS, A., PHAM, H. and MAISONNEUVE, O., "Energy balance of thermoelastic martensite transformation under stress", Nuclear Ingineering and Design, 162, p 1-12, 1996.

[5] CALLEN, H., "Thermodynamics and an introduction to thermostatistics", John Wiley \& sons, 1985.

[6] GERMAIN, P., "Mécanique", Ellipses, Ecole Polytechnique, Tome 1 et 2, 1986.

[7] CHRYSOCHOOS, A. and LOUCHE, H., "An infrared image processing to analyse the calorific effects accompanying strain localisation", Int. J. of Engn. Sci., in press, p 1759-1788, 2000.

[8] CHRYSOCHOOS, A. PHAM H. and MAISONNEUVE O., "Une analyse expérimentale du comportement thermomécanique d'un alliage à mémoire de forme de type Cu-Zn-Al ", C. R. Acad. Sci., t. 316, Série II, p 1031-1036, 1993.

[9] CHADWICK, P. and CREASY, C.F.M., "Modified entropic elasticity of rubberlike materials", J. Mech. Phys. Solids, 32, p 337-357,1984.

[10] OGDEN, R. W., "On the thermoelastic modelling of rubberlike solids", J. of thermal stresses, 15,4,p 533-557, 1992.

[11] SAUREL, J.-L., "Etude thermomécanique d'une famille d'élastomères thermoplastiques", Thesis, Univ. Montpellier II, (1999).

[12] CHRYSOCHOOS, A., LOUCHE, H., MURACCIOLE, J.-M., NEMOZ-GAILLARD M. and WATTRISSE, B "Experimental analysis of localization mechanisms in steels by infrared and speckle image processing", Kluwer Ed., IUTAM symposium on Photomechanics, Poitiers, $p$ 313-320, 1998. 


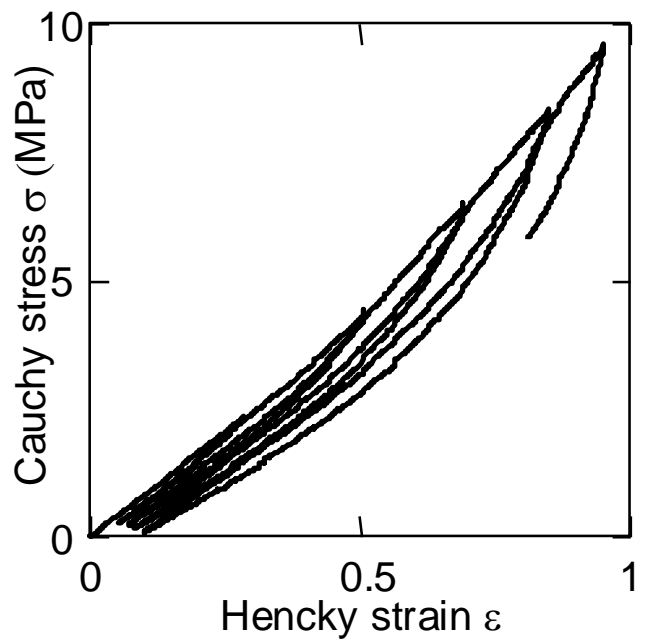

Figure 1: Stress-strain diagram ; Pbax 2533 ; after [11]

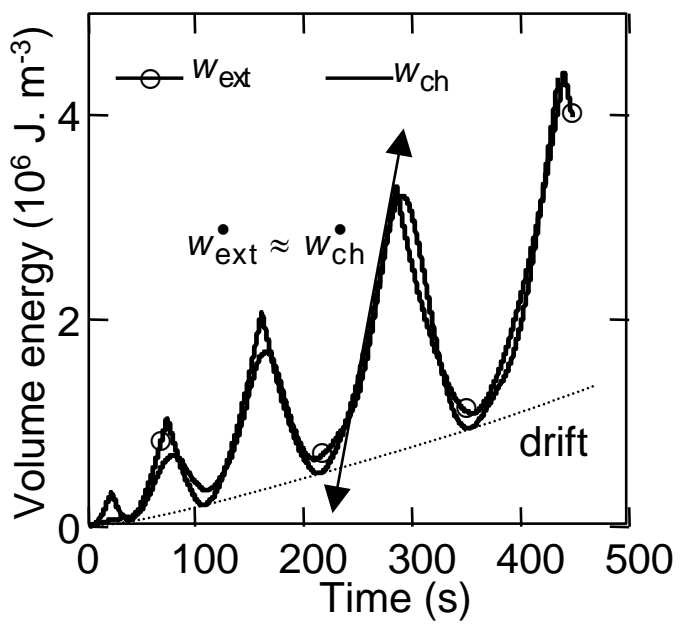

Figure 2: Energy balance ; Pbax 2533; after [11]

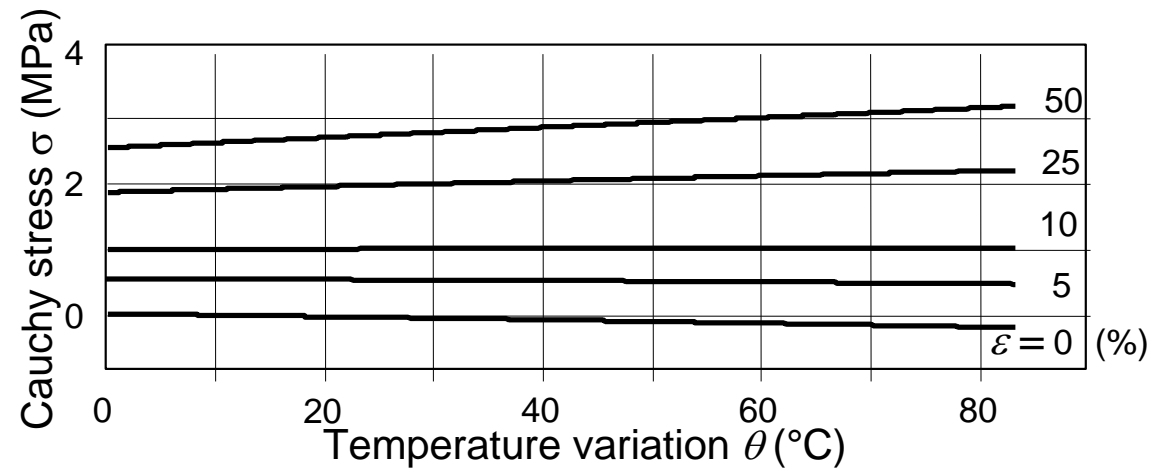

Figure 3 : Simulated "thermoelastic inversion effect" 